\title{
Acquired pulmonary stenosis and pulmonary artery compression
}

\author{
J. H. GOUGH, R. G. GOLD, AND R. V . G I BSON \\ From Brompton Hospital, London, S.W.3
}

\begin{abstract}
Three patients suffering from acquired pulmonary stenosis are described resulting from compression of the main pulmonary artery by anterior mediastinal teratoma, Hodgkin's disease, and aneurysm of the ascending aorta, respectively; also four others who had compression of the right or left primary branch of the pulmonary artery. The pulmonary artery compression was demonstrated angiographically in every case, and in the patient with right and left pulmonary artery compression the main pulmonary artery pressure tracing was characteristic of bilateral pulmonary artery stenosis. Cardiac catheterization and selective angiography are indicated in patients who have evidence of right ventricular outflow obstruction to determine the site and severity of the obstruction and to differentiate between extrinsic compression and intrinsic stenosis.
\end{abstract}

There are two main types of acquired pulmonary stenosis ; both are rare. The first is due to intrinsic disease of the pulmonary valve itself. The second results from extrinsic compression of the pulmonary artery and it is this with which we are concerned here. We believe it to be more common than has been realized, mainly because angiographic studies are not usually carried out on these patients.

In this paper we report three cases of acquired compression of the main pulmonary artery and four of compression of its right or left primary branch. All patients have been studied by right heart catheterization and angiography at Brompton Hospital during the past four years.

\section{CASE REPORTS}

CASE 1 (E. T.) A completely symptom-free girl of 15 years was found on routine chest radiography to have a mass at the left hilum.

On examination there was a loud pulmonary systolic ejection murmur and thrill and a single second sound. Abnormal pulsation was palpable in the second and third left intercostal spaces near the sternal edge. The electrocardiogram showed a mean frontal QRS vector of $+110^{\circ}$ and suggested mild right ventricular hypertrophy. Chest radiography showed an oval mass in the left hilar region which measured $10 \mathrm{~cm}$. in its long diameter and was continuous with the mediastinal shadow (Fig. 1). In the lateral view this mass lay anteriorly and contained a fleck of calcification. The heart appeared normal.

Cardiac catheterization showed a right ventricular pressure of $90 / 0 \mathrm{~mm}$. $\mathrm{Hg}$. and a right pulmonary artery pressure of $17 / 3 \mathrm{~mm}$. $\mathrm{Hg}$. The left pulmonary artery could not be entered. The cardiac output was $4.0 \mathrm{l}$./min. and pulmonary vascular resistance 1.7 units.

An angiocardiogram showed that the mass was compressing and displacing the pulmonary infundibulum and main pulmonary artery downwards and backwards. The mass itself did not opacify (Figs 2 and 3 ).

Left thoracotomy revealed a smooth, pale yellow, cystic mass, approximately $10 \times 7.5 \mathrm{~cm}$., protruding from the mediastinum towards the left. Medially and inferiorly the mass contained a soft cystic area which rested on the pericardium over the region of the pulmonary valve, and a firmer more solid bar of tumour extended backwards from this above and lateral to the pulmonary trunk. Histological examination confirmed that the tumour was a benign teratoma arising in relation to the thymus.

Following removal of the tumour the patient made an uneventful recovery and when seen 16 months later she was symptom-free. The second sound was normal and there was only a very soft pulmonary systolic ejection murmur. The electrocardiogram was within normal limits, with a mean frontal QRS vector of $+90^{\circ}$.

CASE 2 (V.C.) A 22-year-old girl had a history of dyspnoea on exertion for six months with pleuritic pain in the left chest and attacks of dull aching in the upper chest lasting up to 24 hours. She had lost 1 st. $(6 \cdot 3 \mathrm{~kg}$.) in weight in the past two years.

Examination revealed a thin girl with a normal pulse and blood pressure of $100 / 70 \mathrm{~mm}$. $\mathrm{Hg}$. There was a pulsatile prominence in the left chest anteriorly, maximal over the left second and third ribs in the mid-clavicular line. A $4-\mathrm{cm}$. a-wave was 


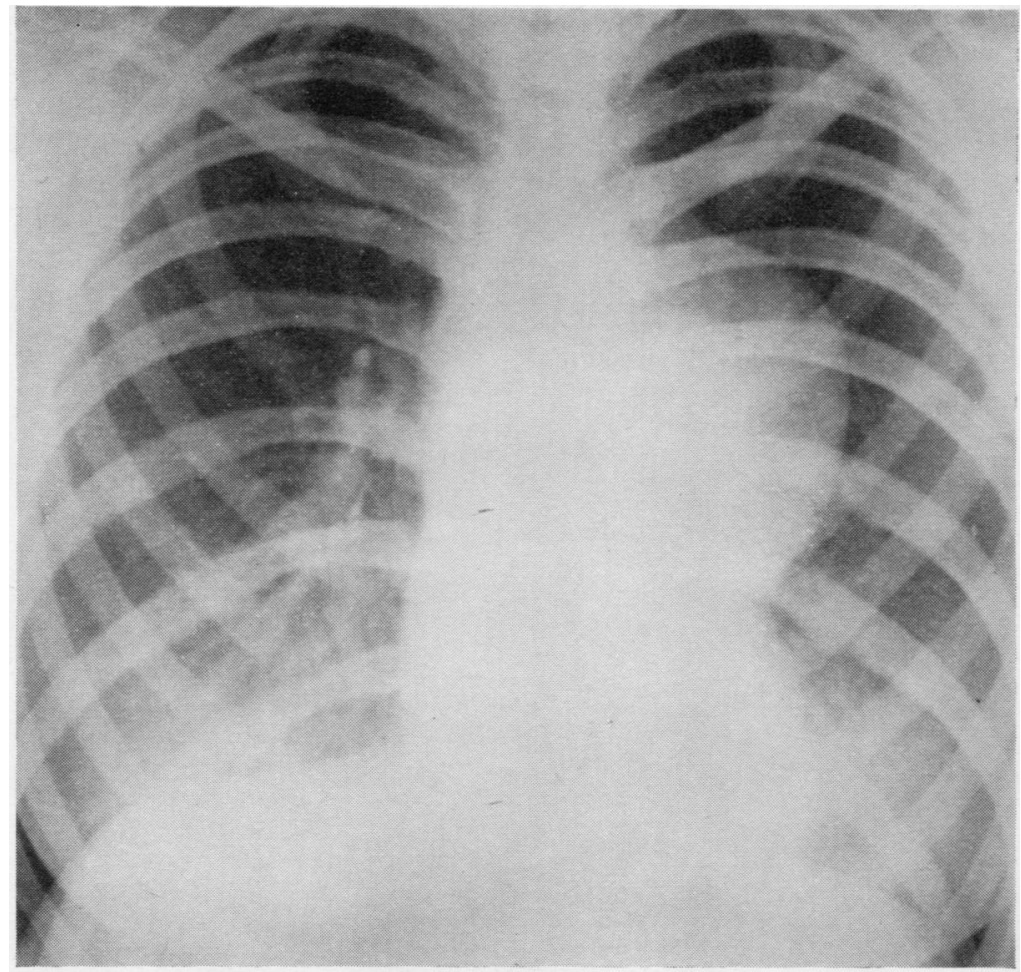

FIG. 1. Case 1. Chest radiograph showing mass in the left hilar region.

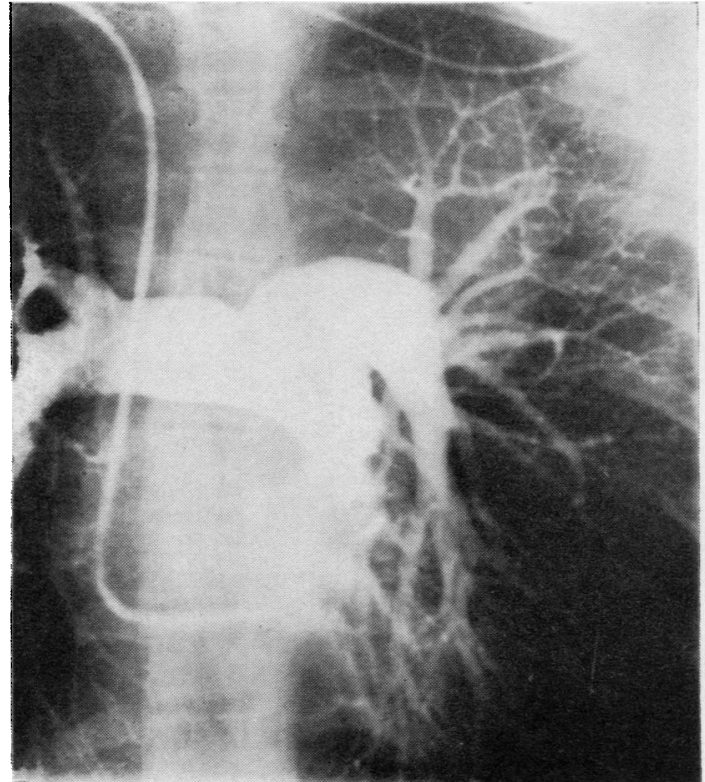

FIG. 2

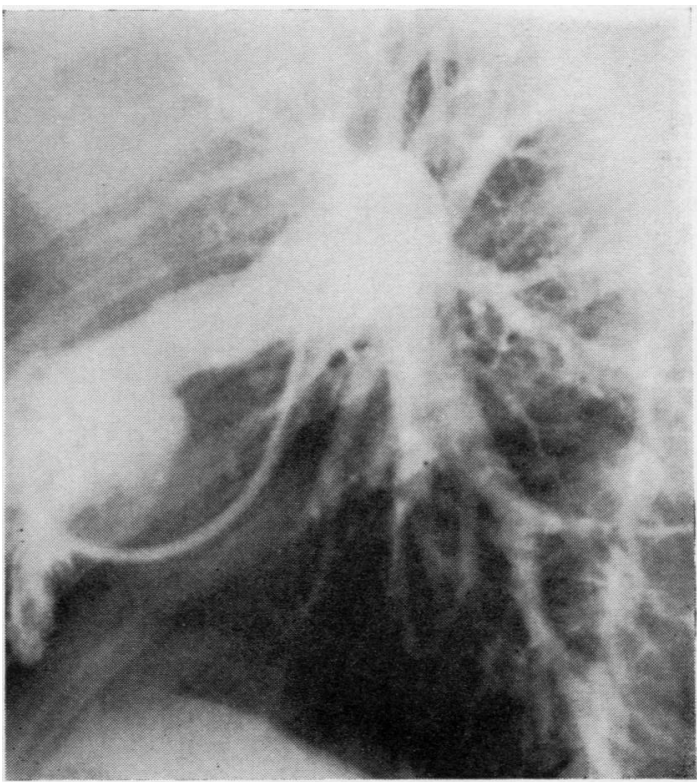

FIG. 3

FIGS 2 and 3. Case 1. A.P. and lateral angiograms (right ventricular injection). The main pulmonary artery is compressed and displaced downwards and backwards by the tumour. 
present in the jugular venous pulse and a moderate right ventricular impulse was palpable at the left sternal edge. A moderate pulmonary systolic ejection murmur was heard at the left sternal edge, with no ejection click. The pulmonary component of the second sound was diminished and delayed. The lungs were clinically normal. An electrocardiogram showed moderate right ventricular hypertrophy with a mean frontal QRS vector of $+130^{\circ}$, a dominant $R$ in lead $\mathrm{V} 1$, and considerable clockwise rotation in the precordial leads. (An electrocardiogram done two years previously had shown a mean frontal QRS vector of $+95^{\circ}$ in an otherwise normal tracing.) Blood examination showed a haemoglobin of $10.8 \mathrm{~g} . / 100 \mathrm{ml}$., M.C.H.C. $29 \%$, a white count of $10,000 /$ c.mm., with $79 \%$ neutrophils, $2 \%$ eosinophils, $13 \%$ lymphocytes. and $6 \%$ monocytes. The erythrocyte sedimentation rate was $55 \mathrm{~mm}$. in one hour.

Chest radiography showed a massive opacity in the left hilar region which in the lateral view lay anteriorly (Fig. 4). There was a small left pleural effusion. The pulmonary vessels on the left were reduced in calibre while those on the right appeared normal. The heart shadow was normal.

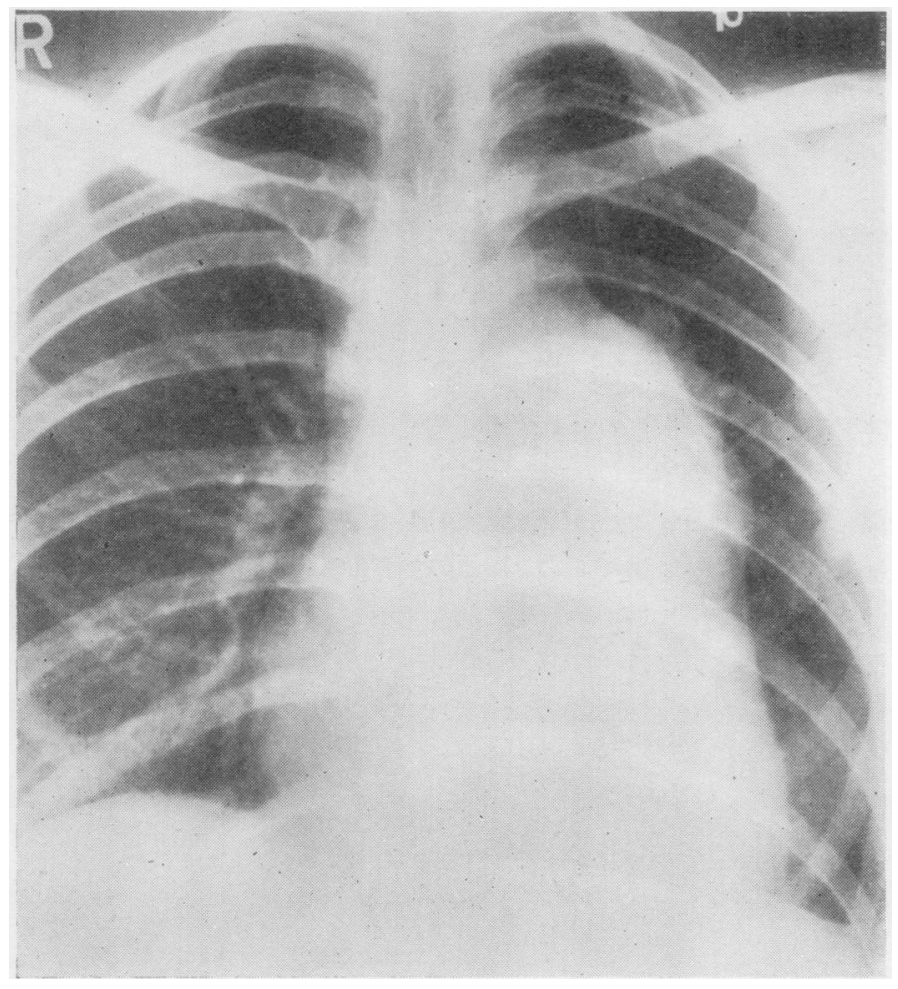

FIG. 4. Case 2. Chest radiograph showing massive opacity in the left hilar region extending downwards. The peripheral pulmonary vessels are small on the left side compared with those on the right.

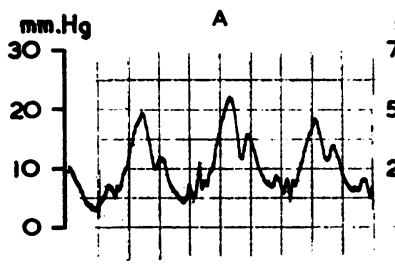

RPA

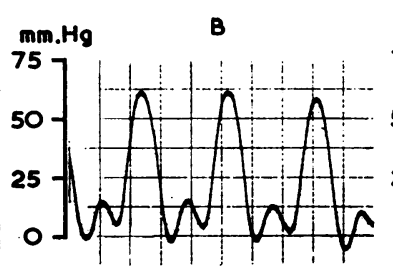

MPA

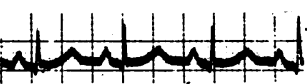

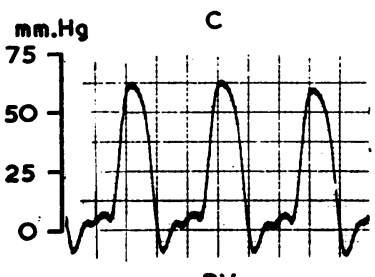

RV

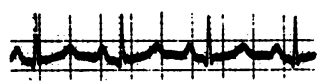

FIG. 5. Case 2. Pressure records obtained at cardiac catheterization from $(A)$ right pulmonary artery $(R P A),(B)$ main pulmonary artery $(M P A)$, and $(C)$ right ventricle $(R V)$.

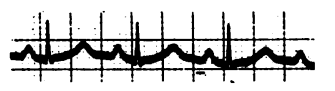


Cardiac catheterization revealed a right ventricular pressure of $70 / 0 \mathrm{~mm}$. $\mathrm{Hg}$ with a main pulmonary artery pressure of $60 / 2 \mathrm{~mm}$. $\mathrm{Hg}$ and right and left pulmonary artery pressures of $20 / 5$ and $10 / 5 \mathrm{~mm}$. $\mathrm{Hg}$ respectively. The wave form of the main pulmonary artery tracing showed a steep rise and fall of pressure with a large dicrotic notch and a low diastolic pressure (Fig. 5).

An angiocardiogram showed that the right ventricular outflow tract and the whole of the main pulmonary artery were indented from above and considerably narrowed. The left pulmonary artery showed severe narrowing which extended distally to the origin of the first upper lobe branch. The distal branches on the left were all reduced in calibre. The right pulmonary artery was also slightly narrowed at its origin (Figs 6 and 7).

Mediastinal biopsy revealed a hard, white, gritty tumour mass presenting in the left anterior third intercostal space. Histological examination showed Hodgkin's disease. The patient received a course of deep radiotherapy with considerable subjective improvement, a decrease in the a-wave in the jugular venous pulse, and a return to normal in the movement and intensity of the pulmonary component of the second sound, though the systolic ejection murmur persisted unchanged. An electrocardiogram one year later still showed a mean frontal QRS vector of

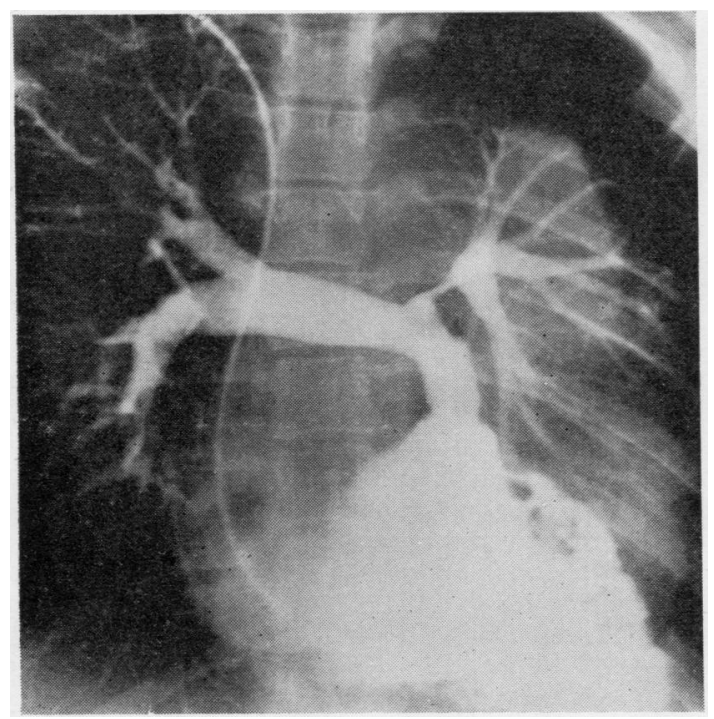

FIG. 6

Figs 6 and 7. Case 2. A.P. and lateral angiograms (right ventricular injection). The right ventricular outflow tract and main pulmonary artery are narrowed and indented from above. There is very severe narrowing of the proximal left pulmonary artery. The peripheral branches on the left are reduced in calibre. $+110^{\circ}$ but was otherwise normal, and the patient was well and back at work. Chest radiographs taken three and a half years after she had started treatment showed some displacement of the heart to the left and a little linear shadowing in the left upper zone, probably due to fibrosis following radiotherapy. The pulmonary vessels on the left were still smal! compared with those on the right.

CASE 3 (E. D.) A 52-year-old man, who is fully reported elsewhere (Yacoub, Braimbridge, and Gold, 1966), presented with an abnormal systolic impulse in the second left intercostal space at the left sternal edge, an inspiratory pulmonary ejection murmur, and delay and diminution of the pulmonary component of the second sound. An electrocardiogram showed evidence of ischaemic heart disease. Chest radiography revealed a mass, $9 \times 6 \mathrm{~cm}$., to the left of and in front of the ascending aorta with flecks of linear calcification in it. At right heart catheterization there was a $24 \mathrm{~mm}$. Hg systolic gradient between the pulmonary artery and the right ventricle, and the right ventricular pressure was $44 \mathrm{~mm}$. $\mathrm{Hg}$.

An angiogram (right ventricular injection) showed compression and distortion of the right ventricular outflow tract, pulmonary valve, and main pulmonary artery, which were displaced downwards and backwards by a saccular aortic aneurysm, $8 \times 5.5 \mathrm{~cm}$.,

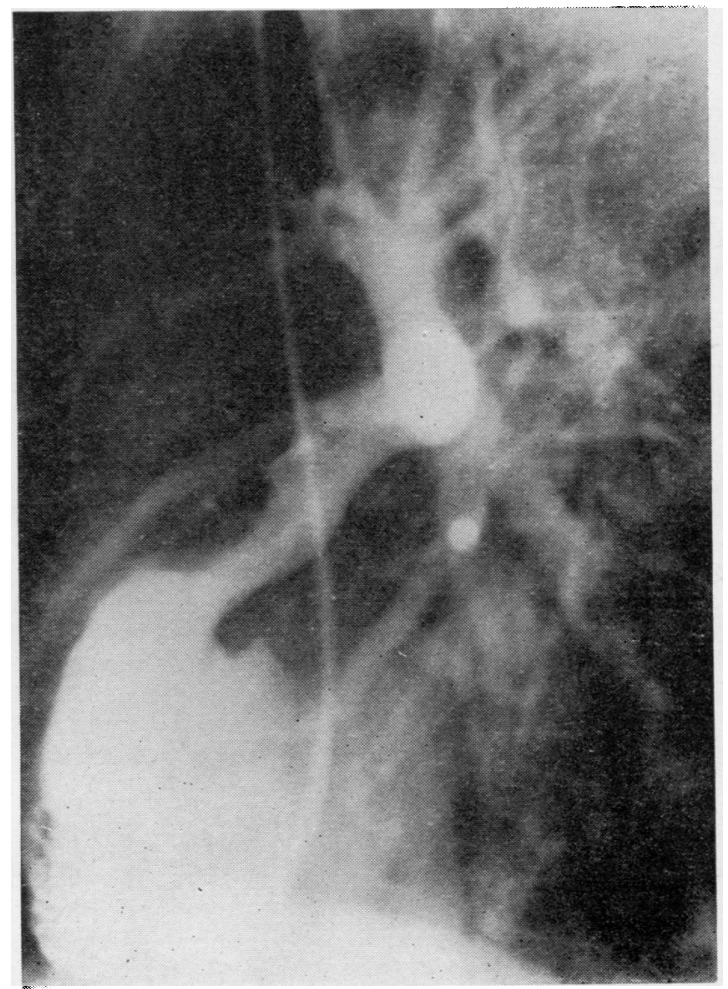

FIG. 7 


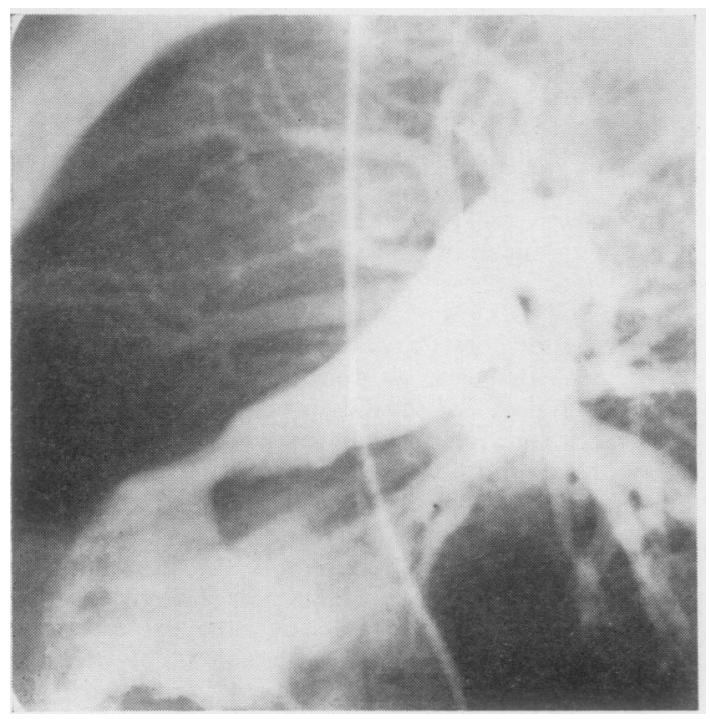

FIG. 8. Case 3. Lateral angiogram (right ventricular injection) shows compression and distortion of the right ventricular outflow tract and main pulmonary artery.

arising from the upper part of the ascending aorta (Fig. 8). This contained much clot. Repair of the aneurysm was followed by complete recovery and disappearance of all signs of pulmonary stenosis.

CASE 4 (W. M.) A man aged 31 had had a dry cough for many years, but about six months before his admission it had become productive of green sputum. He had also noticed progressive dyspnoea on exertion. On examination the cardiovascular system was normal. Chest radiography showed prominence of the ascending aorta and also prominence of the aorta to the left of the mid-line down to the level of the hilum (Fig. 9). No aortic calcification was visible. The blood Wassermann reaction and Kahn test were positive.

At bronchoscopy a pulsatile mass was encroaching on the trachea from its left anterior aspect, reducing its lumen by half. The mass narrowed the left main bronchus to a slit.

An angiogram (pulmonary artery injection). showed slight dilatation of the ascending aorta. There was a saccular aneurysm of the aortic arch, extending from the origin of the innominate artery to just beyond the origin of the left subclavian artery (Fig. 10). This was displacing the proximal part of the right pulmonary artery downwards and narrowing it slightly (Fig. 11).

The patient was treated initially with bismuth and penicillin, but it was felt that operation was advisable in view of the otherwise poor prognosis. At operation, using Melrose perfusion with profound hypothermia, the aorta was found to be aneurysmally dilated from a point $2 \mathrm{~cm}$. above the valve to the left subclavian artery. It was compressing the trachen and left main bronchus. The aneurysm was excited and a Tefion $\overrightarrow{\vec{F}}$ graft was inserted, but at the end of the procedure $\stackrel{\oplus}{+}$ the heart action became feeble and incapable of $\bar{C}$ sustaining an adequate blood pressure. Attempts at $\overline{\bar{\sigma}}$ resuscitation were unsuccessful.

CASE 5 (A. S.) A man aged 41 years complained of dyspnoea on exertion, fatigue, and hoarseness for a few weeks, and he had lost 2 st. $(12.7 \mathrm{~kg}$.) in weight. $\overrightarrow{0}$ On examination there were no abnormal signs in the chest or cardiovascular system.

The chest radiograph revealed a mass near the aortic knuckle, about $7 \mathrm{~cm}$. in diameter (Fig. 12). $\underset{\times}{\vec{x}}$ Lateral tomographs showed that this lay lateral to the upper part of the descending thoracic aorta and $N$ also extended anteriorly.

Sputum was negative for neoplastic cells and it was uncertain whether the mass was an aortic aneurysm or a tumour. A pulmonary arteriogram was performed and this showed severe narrowing of $\overrightarrow{-}$ the proximal part of the left pulmonary artery (Fig. 13). The main pulmonary artery and its right branch $₹$ were normal, as was the aorta. All the peripheral $\overrightarrow{0}$ branches of the left pulmonary artery were reduced in calibre, though this was not apparent on the plain films.

Bronchoscopy showed left vocal cord paralysis. A tumour was visible in the left main bronchus, extend- $\bar{\partial}$ ing to within $0.5 \mathrm{~cm}$. of the carina. Biopsy showed a squamous-cell bronchial carcinoma.

The patient was treated with radiotherapy. However, his condition deteriorated and he died nine $\overrightarrow{0}$ months after starting treatment. At necropsy the tumour appeared to be arising from the left main bronchus and was extending into both the upper and lower lobes of the left lung. It was invading the oesophagus, vertebral muscles, and chest wall.

CASE 6 (E. J.) A woman aged 64 complained of $\dot{\sigma}$ dyspnoea on exertion, productive cough, and loss of 3 weight for about one month. On examination there were expiratory rhonchi on both sides of the chest, but more on the left. The cardiovascular system was normal.

Chest radiographs and tomograms showed a mass $\frac{D}{O}$ in the left hilar region close to the proximal part of the left pulmonary artery and extending backwards. $N$ The peripheral lung vessels were smaller on the left ${ }^{\mathrm{T}}$ than on the right.

Bronchoscopy was normal and no neoplastic cells could be found either in the sputum or in trap $\sigma$ specimens from the left bronchial tree. A pulmonary

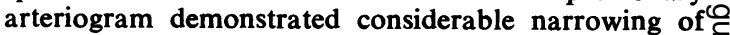
the left pulmonary artery, starting proximal to the $\mathbb{E}$ origin of the upper lobe branches and extending distally to involve the origins of two lower lobe basal $\square$ segmental branches. The lateral margin of the upper descending thoracic aorta was indented at the level $\overparen{D}$ of the left hilar mass.

At thoracotomy growth had ulcerated through the $\frac{\Omega}{\sigma}$ mediastinal pleura and was infiltrating the wall of the upper descending aorta. The sub-aortic fossa was $ᄋ$ 

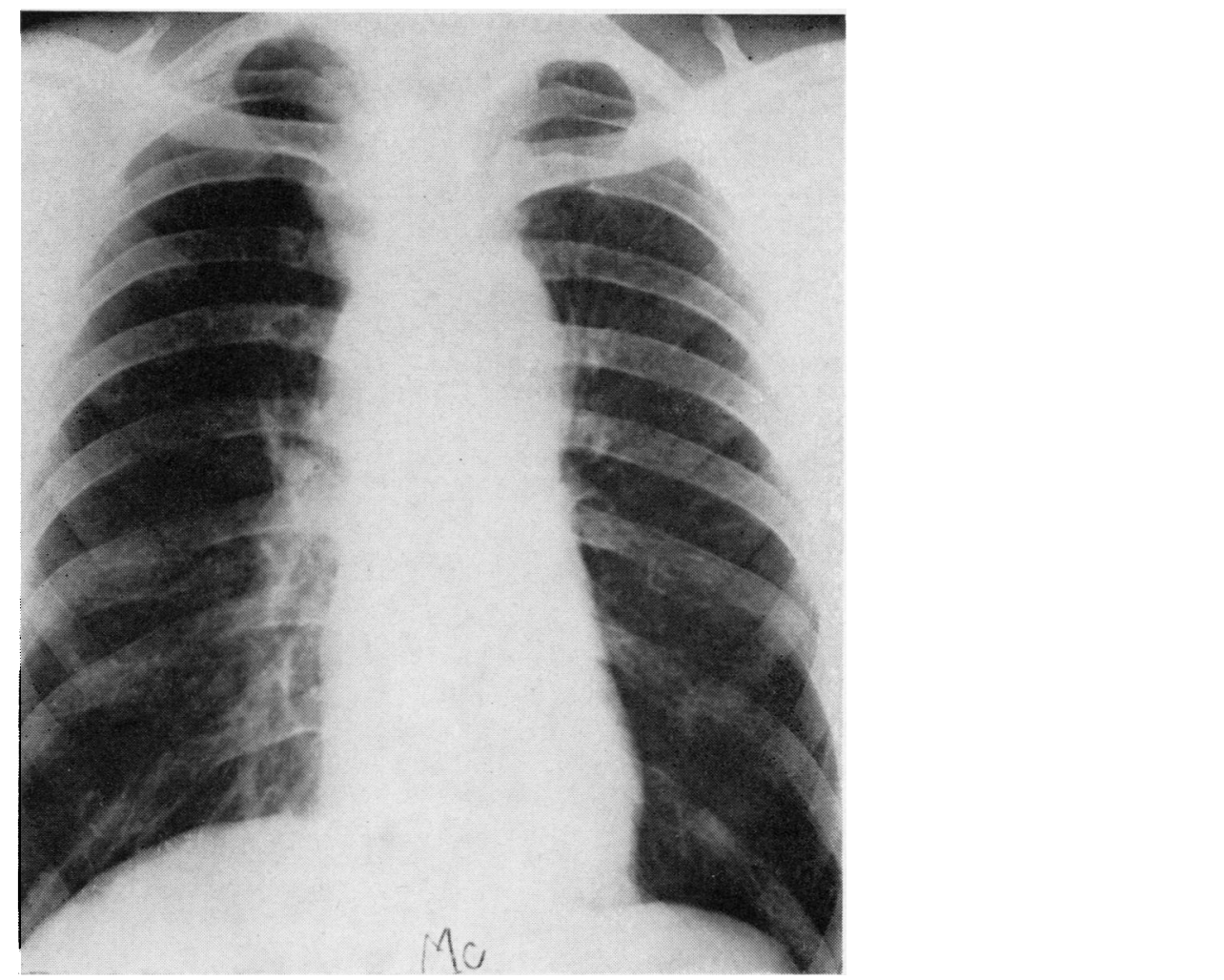

FIG. 9. Case 4. Chest radiograph shows prominence of the aorta on both sides of the mid-line.

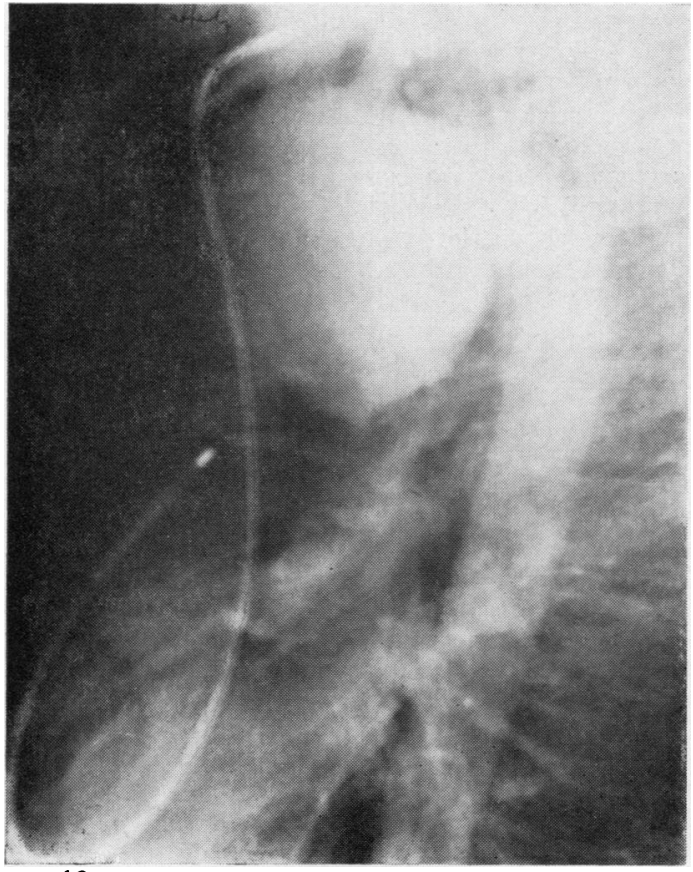

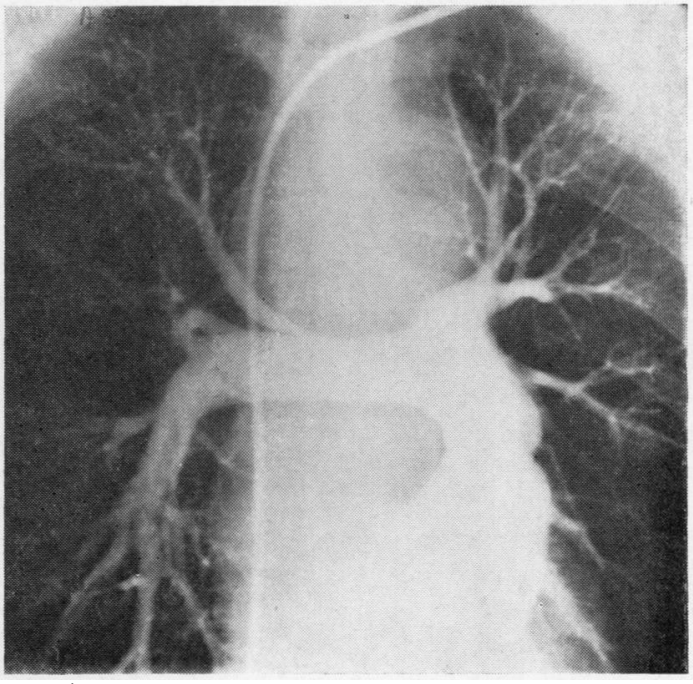

F:G. 1:

FIGS 10 and 11. Case 4. Lateral and A.P. angiograms (pulmonary artery injection) showing saccular aneurysm of the aortic arch. This is displacing the right pulmonary artery downwards and narrowing it slightly. 
FIG. 12. Case 5. Chest radiograph showing mass near the aortic knuckle extending downwards.
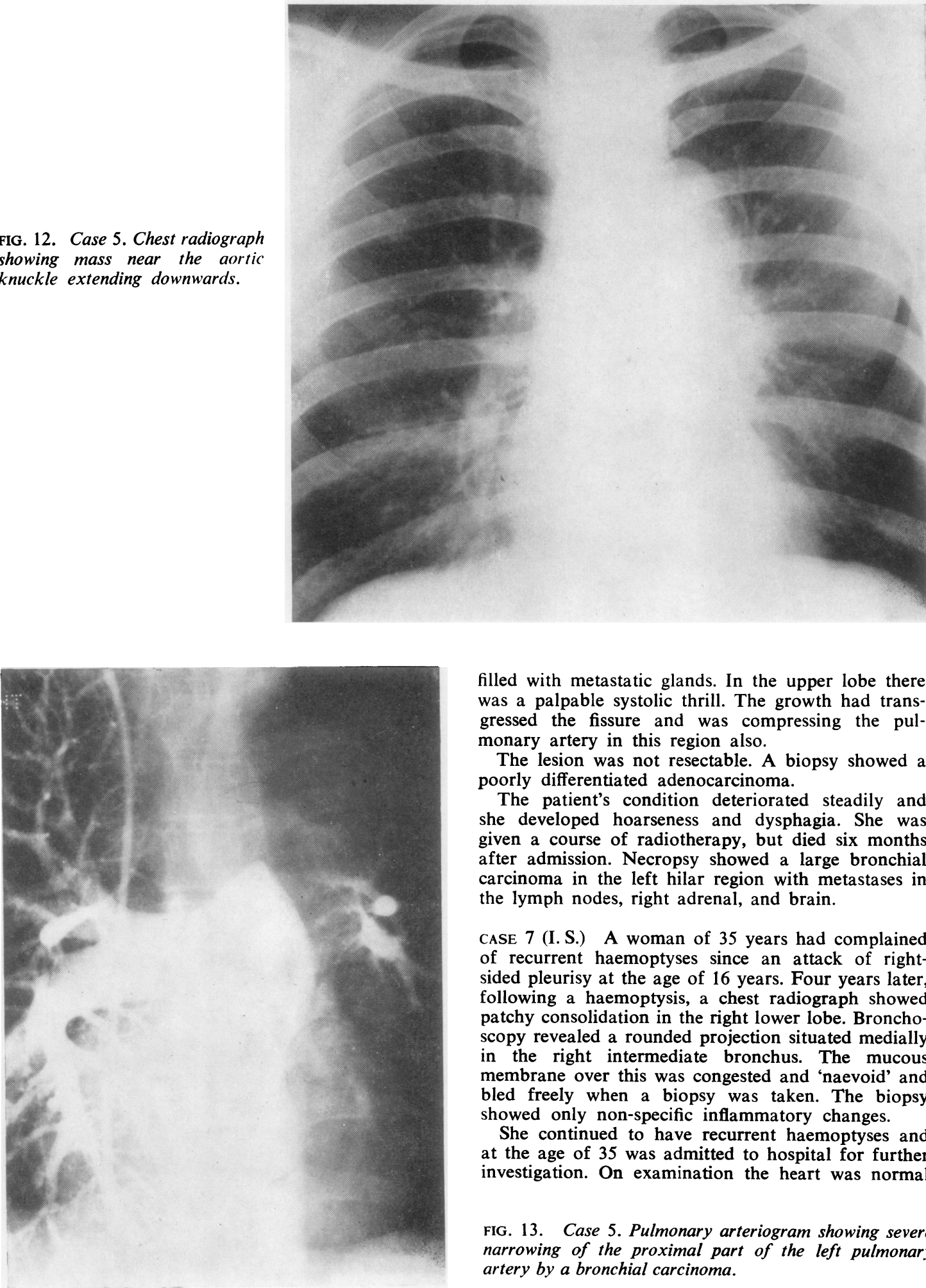

filled with metastatic glands. In the upper lobe there was a palpable systolic thrill. The growth had transgressed the fissure and was compressing the pulmonary artery in this region also.

The lesion was not resectable. A biopsy showed a poorly differentiated adenocarcinoma.

The patient's condition deteriorated steadily and $\underset{x}{0}$ she developed hoarseness and dysphagia. She was given a course of radiotherapy, but died six months after admission. Necropsy showed a large bronchial $\delta$ carcinoma in the left hilar region with metastases in the lymph nodes, right adrenal, and brain.

CASE 7 (I.S.) A woman of 35 years had complained of recurrent haemoptyses since an attack of rightsided pleurisy at the age of 16 years. Four years later, $N$ following a haemoptysis, a chest radiograph showed patchy consolidation in the right lower lobe. Broncho- $\mathrm{O}$ scopy revealed a rounded projection situated medially $\mathrm{\omega}$ in the right intermediate bronchus. The mucous $\sigma$ membrane over this was congested and 'naevoid' and bled freely when a biopsy was taken. The biopsy showed only non-specific inflammatory changes.

She continued to have recurrent haemoptyses and? at the age of 35 was admitted to hospital for further investigation. On examination the heart was normal $\underset{\mathbb{D}}{\circ}$

FIG. 13. Case 5. Pulmonary arteriogram showing severe narrowing of the proximal part of the left pulmonary< artery by a bronchial carcinoma. 


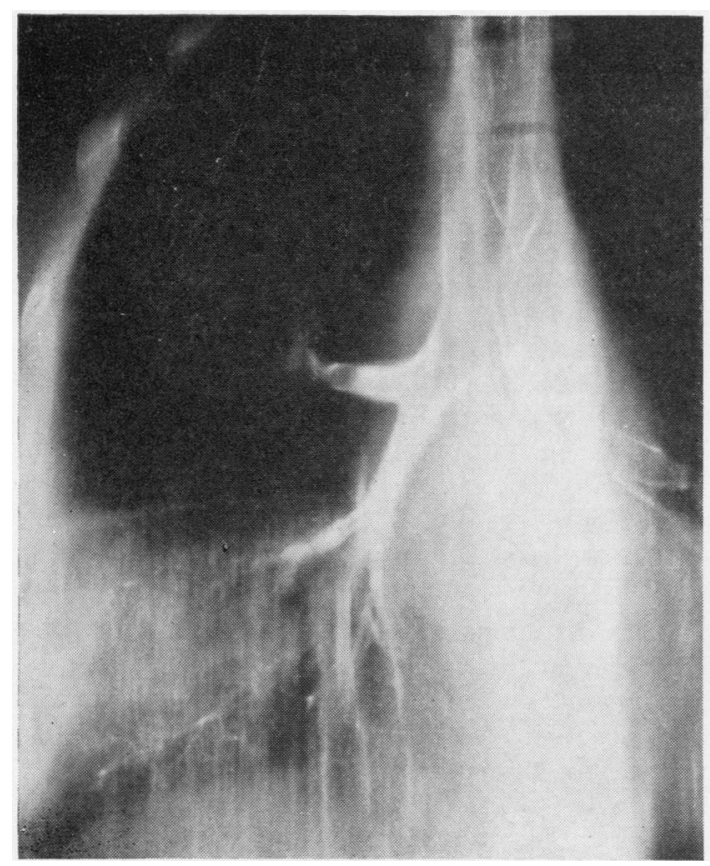

FIG. 14. Case 7. Tomo-bronchogram showing slight irregularity of the medial wall of the right intermediate bronchus. Medial to this bronchus there is an area of calcification.

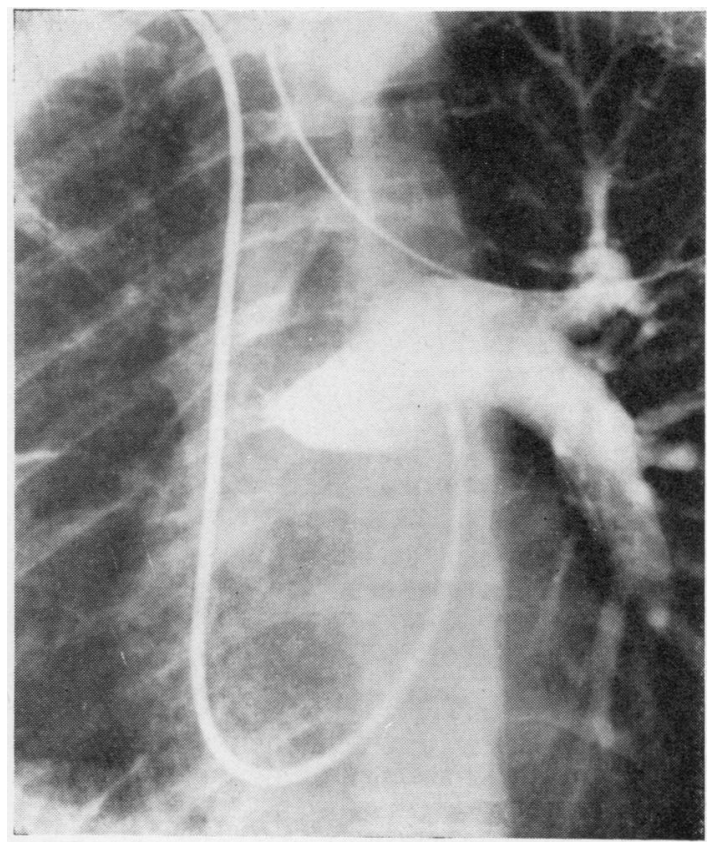

FIG. 16. Case 7. Pulmonary arteriogram showing a complete block of the right pulmonary artery.

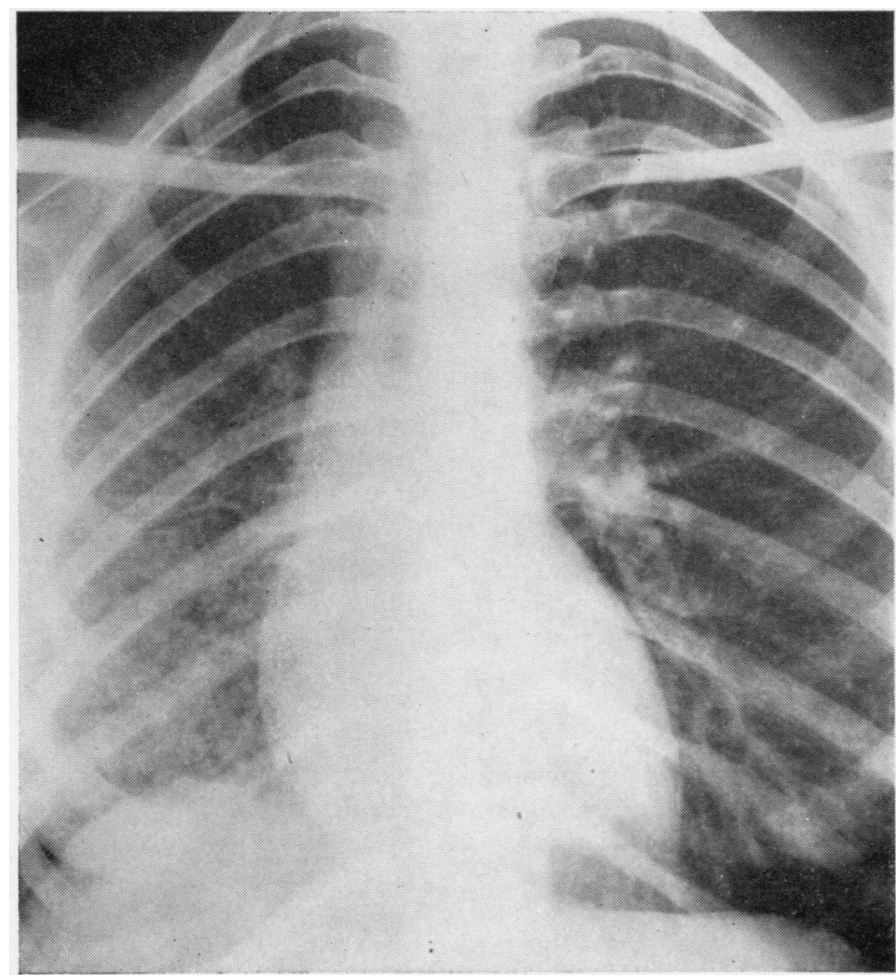

FIG. 15. Case 7. Chest radiograph. There is diffuse shadowing in the right mid and lower zones, and complete absence of the normal vascular pattern throughout the right lung. 
except for a soft pulmonary systolic ejection murmur and an expiratory pulmonary ejection click. The trachea was deviated slightly to the right and there was diminished chest movement on the right side with some dullness to percussion and diminished air entry over the right lower lobe. The Mantoux test was strongly positive but sputum cultures for tubercle bacilli were negative.

A tomo-bronchogram demonstrated irregularity of the medial wall of the right intermediate bronchus. Just medial to this bronchus there was an area of irregular calcification (Fig. 14). The diffuse shadowing, mainly in the right lower lobe, persisted and there was absence of the normal vascular pattern throughout the right lung (Fig. 15).

Cardiac catheterization revealed normal resting right heart pressures, but exercise produced a rise in pulmonary artery pressure from $25 / 6$ to $45 / 14 \mathrm{~mm}$. $\mathrm{Hg}$. The catheter could easily be passed into the left pulmonary artery but would not pass beyond the region of the right main bronchus in the right pulmonary artery.

A pulmonary arteriogram showed that about $2 \mathrm{~cm}$. from its origin, i.e., just before the point where it crossed the right bronchus, the right pulmonary artery tapered rapidly and was completely blocked (Fig. 16). The pulmonary trunk and left pulmonary arterial tree were normal.

At thoracotomy the right lung was adherent to the parietal pleura. Extrapleural stripping was performed. Many vessels entered the pleura from the parietes. At the hilum a hard mass was palpable surrounding the right main bronchus and right pulmonary artery and fixed to the posterior wall of the superior vena cava. The pulmonary trunk was free. Many of the bronchial vessels entering the lung from the hilum were divided to reduce the risk of haemoptysis.

- A biopsy was taken from the mass. This contained areas of calcification and was reported as being probably tuberculous.

During the three years since operation the patient has had no more haemoptyses and remains symptomfree.

\section{DISCUSSION}

Acquired pulmonary stenosis due to extrinsic compression of the main pulmonary artery is rare. References to it in the literature are scanty and most authors describe only a single case. Thus, in 1962, Babcock, Judge, and Bookstein, in a paper describing a case of pulmonary stenosis due to lymphoblastoma of the anterior mediastinal glands, wrote, 'Mediastinal neoplasms rarely obstruct the great arteries. To our knowledge only five cases of pulmonary stenosis produced by extrinsic compression have been reported'. Similarly, a year later, Schrire, Beck, and Barnard (1963) could find in the literature only 11 cases-to which they added one more-of aortic aneurysm causing pulmonary stenosis. In none $\overrightarrow{\vec{c}}$ of these 12 cases was the stenosis demonstrated angiographically.

Other recognized causes of this type of pulmo- $\frac{\overline{\bar{N}}}{\vec{S}}$ nary stenosis are mediastinal teratoma (Fry, $\mathbb{\otimes}$ Klein, and Barton, 1955), thymic tumours (Winter, 1958), bronchial carcinoma (Abbey Smith, 1957), mesothelioma of the pericardium $\vec{\circ}$ (Waldhausen, Lombardo, and Morrow, 1957), and $\overrightarrow{\vec{\omega}}$ rheumatic pericarditis (Gouley, 1937). Compression of the right or left branch of the pulmonary $\vec{x}$ artery, without involvement of the pulmonary trunk, is more common.

Three of our patients exhibited clinical features $\stackrel{+}{\omega}$ of pulmonary stenosis. Pulmonary systolic ejec- $\infty$ tion murmurs were prominent in all of these, and $\frac{}{5}$ in one patient (case 1) a systolic thrill was alsopresent. In this patient the second heart sound $\subseteq$ was single; in the other two (cases 2 and 3 ) the pulmonary component was diminished and $\vec{\oplus}$ delayed. None of these patients had a pulmonary ejection click. Absence of the latter in a patient with signs of pulmonary stenosis suggested that the stenosis was not valvar, but there was no clinical evidence in these cases to indicate that the obstruction was due to extrinsic compression $\frac{\circ}{\Phi}$ of the pulmonary outflow tract. Identical physical $\stackrel{\varrho}{\rightarrow}$ signs could have been recorded in congenital $\overrightarrow{0}$ infundibular or supravalvar pulmonary stenosis. 3 Cardiac catheterization provided a measurement of the severity of the obstruction. The right ventricular pressures in these three patients ranged $\vec{\partial}$ from 44 to $99 \mathrm{~mm} . \mathrm{Hg}$, and the gradient between $\frac{0}{0}$ the right ventricle and main pulmonary artery varied from 10 to $73 \mathrm{~mm}$. $\mathrm{Hg}$. In case 2 the pres- $\frac{3}{3}$ sure records showed that the major obstruction was at the origins of the right and left pulmonary arteries, but in the other two patients pressure records were inconclusive in localizing the obstruction, as a single pressure change, indistinguishable $\frac{D}{0}$ from that obtained in pulmonary valve stenosis, occurred on withdrawal of the catheter from the or pulmonary artery to the right ventricle. For this reason we consider angiography to be essential in differentiating between extrinsic compression $\omega$ and intrinsic obstruction of the pulmonary artery and infundibulum.

Agustsson, Arcilla, Gasul, Bicoff, Nassif, and Lendrum (1962), in cases of bilateral pulmonary ${ }_{T}^{+}$ artery stenosis, described a characteristic wave- $-\overrightarrow{0}$ form in the pressure records taken from the main $\vec{\Phi}$ pulmonary artery, and D'Cruz and his colleagues $\frac{?}{\mathbb{D}}$ observed this type of pressure record in most of $\varrho$ 52 patients with bilateral pulmonary artery stenosis (D’Cruz, Agustsson, Bicoff, Weinberg, $̊$ 
and Arcilla, 1964). We have been unable to find in the literature any example of extrinsic compression of both pulmonary arteries as a cause for this type of pressure tracing and believe that our patient (case 2, Fig. 5) is the first to be described. The murmur and wide splitting of the second sound were strikingly similar to those noted by D'Cruz et al.

In the four patients (cases 4 to 7) in whom the main pulmonary trunk was not involved, no clinical evidence of pulmonary stenosis or of pulmonary artery branch stenosis was present, although at operation on case 6 a thrill was felt over the upper lobe of the left lung. Cardiac catheterization revealed no pressure gradients in these patients, the compression of a pulmonary artery being demonstrated solely by angiography.

In the three patients showing clinical evidence of pulmonary stenosis, relief of the obstruction was followed by a return to normal in the behaviour of the second sound, though some degree of systolic murmur persisted in two of them. In the two patients with electrocardiographic evidence of right ventricular hypertrophy, this disappeared following relief of the obstruction.
We wish to thank Mr. M. Paneth, who operated on cases 2, 4, and 6, and Mr. O. S. Tubbs, who operated on cases 1 and 7 , for permission to use their operation notes.

\section{REFERENCES}

Agustsson, M. H., Arcilla, R. A., Gasul, B. M., Bicoff, J. P., Nassif, S. I., and Lendrum, B. L.' (1962). The diagnosis of bilateral stenosis of the primary pulmonary artery branches based on characteristic pulmonary trunk pressure curves. Circulation, 26, 421 .

Babcock, K. B., Judge, R. D., and Bookstein, J. J. (1962). Acquired pulmonic stenosis. Report of a case caused by mediastinal neoplasm. Ibid., 26, 931.

D'Cruz, I. A., Agustsson, M. H., Bicoff, J. P., Weinberg, M., and Arcilla, R. A. (1964). Stenotic lesions of the pulmonary arteries. Amer. J. Cardiol., 13, 441.

Fry, W., Klein, C. L., and Barton, H. C. (1955). Malignant mediastinal teratoma simulating cardiovascular disease. Dis. Chest, 27, 537.

Gouley, B. A. (1937). Constriction of the pulmonary artery by adhesive pericarditis. Amer. Heart J., 13, 470.

Schrire, V., Beck, W., and Barnard, C. N. (1963). Aneurysm of the ascending aorta obstructing right ventricular outflow and producing severe 'pulmonary stenosis'. Ibid., 65, 396.

Smith, R. Abbey (1957). The results of raising the resectability rate in operations for lung carcinoma. Thorax, 12, 79.

Waldhausen, J. A., Lombardo, C. R., and Morrow, A. G. (1957). Pulmonic stenosis due to compression of the pulmonary artery by an intrapericardial tumor. J. thorac. Surg., 37, 679.

Winter, B. (1958). Pulmonic stenosis produced by compression of heart by anterior mediastinal tumor. Amer. Heart J., 55, 18.

Yacoub, M. H., Braimbridge, M. V., and Gold, R. G. (1966). Aneurysm of the ascending aorta presenting with pulmonary stenosis. Thorax, 21, 236. 